\title{
Small bowel tumor: Surgeon's perspectives in a tertiary health care centre
}

\author{
Sadaf Ali, Khalid Bashir, Feroz Khan, Jaswinder Singh Soddi, \\ Feroz Shaheen, Muneer Ahmed, Omar Shah, Showkat Zargar
}

\begin{abstract}
Introduction. Small bowel cancers are rare. The past decade has seen significant advances in the evaluation of the small bowel once considered to be blind to evaluate. Accumulation of data regarding their clinical presentation, pathologic features, prognostic factors, treatment modalities, and outcome is difficult.

Methods. This is a retrospective study of 26 patients with small bowel cancers treated at the department of surgical gastroenterology at a tertiary health care centre in Kashmir valley of India. The records were analyzed in terms of clinical presentation, histopathological diagnosis, treatment and follow up (survival) of these small bowel tumors.

Results. The study included 15 males and 11 females. Median age at presentation was 48 years. Most common symptoms were abdominal pain, obscure gastrointestinal bleed, weight loss and sub acute gastrointestinal obstruction in descending order. Ten patients presented with abdominal emergencies. Twenty two patients (84.61\%) had a diagnosis preoperatively with capsule endoscopy or a 64 slice CT enterography. Gastrointestinal stromal tumor was the most common tumor, followed by adenocarcinoma, lymphoma and neuroendocrine tumors. Curative $R 0$ resection was achieved in all but two patients.

Conclusion. Small bowel cancers are difficult to diagnose because of the nonspecific symptoms. Newer tools like capsule endoscopy and CT enterograhy are now a solution to this diagnostic dilemma disease. Survival of small bowel tumors is fairly good if diagnosed timely. Different pathological types of small bowel tumors behave differently.
\end{abstract}

\section{Introduction:}

Tumors of the small bowel are relatively rare in incidence. They account for only approximately $3-6 \%$ of all gastrointestinal neoplasm's and 1-3\% of all gastrointestinal malignancy ${ }^{1}$. The distribution of small bowel cancer histology in literature is commonly carcinoid, adenocarcinoma, lymphoma, and gastrointestinal stromal tumor (GIST) ${ }^{2}$. With the advanced understanding of obscure gastrointestinal bleed, small bowel inflammatory diseases, syndromal polyposis and the advent of newer devices to work up the so called dead end or black box area now being seen more clearly. The available tools for small bowel evaluation are capsule endoscopy, push enteroscopy, CT enterography and CT enterocalysis. With this advancement in endoscopy and radiology the extremely rare small intestinal lesions are now being picked up more frequently. Korean national cohort of wireless capsule endoscopy (WCE) which was performed with indications including obscure gastrointestinal bleeding (OGIB), abdominal pain, weight loss and diarrhea picked up small bowel tumors in $4.3 \%$ cases ${ }^{3}$. During the past 10 years, spiral computed tomographic (CT) enterocalysis has evolved into an established modality for the investigation of various small-bowel disorders with an overall sensitivity and specificity in identifying patients with small-bowel lesions were $84.7 \%$ and $96.9 \%$, respectively ${ }^{4}$.

Present study was conducted to review the experience with Small bowel tumors of 26 patients managed at a surgical gastroenterology unit at a tertiary health care centre.

\section{Material and Method:}

Records of prospectively collected data of 26 cases of small tumor managed at a tertiary health care centre was reviewed and analyzed.

Patients were studied for :

- Demographic details

- Presenting signs and symptoms

- Diagnostic workup

- Operative procedures

- Postoperative Complications

- Pathologic diagnosis

- Patients qualifying follow up of minimum of 3 years after diagnosis were included in this study

- Recurrence was defined as biopsy-proven tumor or radiologic evidence of obvious local or distant recurrence. 
Periampullary tumors unless pathologically proven to be arising from duodenum and benign lesions of the small bowel were all excluded.

The tumor staging was done according to the pathological staging of the particular diagnosis as TNM classification for adenoca, Fletcher risk grading for GIST, WHO grading system for neuroendocrine tumors and Ann Arbors adapted grading system for lymphomas.

Radical curative resection was aimed in all patients. Tumors of duodenum had a pylorus preserving pancreatico duodenectomy. Tumors of jejunum and ileum were resected with 10 centimeter proximal and distal margins along with 6-8 $\mathrm{cm}$ deep mesentery.

Operative details were assessed in terms of morbidity and mortality.

Survival duration was measured from the time of diagnosis to the last follow-up evaluation or death. Follow up data was maintained from patient's regular check up scheduled every 3 months. On follow up a detailed history of any recurrence of symptoms and clinical examination were noted by the faculty. A follow up CT was done after 1 year in selective cases. Non complaint patient were called on telephone numbers in records and patient or family members were interviewed.

\section{Results:}

This study included 26 patients with histologically proven small bowel malignancies. They were 15 males $(57.69 \%)$ and 11 females $(42.30 \%)$ patients. The male to female ratio was $1.3: 1$. The median age at presentation of 48 years (range: $21-72$ ).

Most of complaints were nonspecific and these included in decreasing order abdominal pain, obscure gastrointestinal bleed, weight loss, and sub acute intestinal obstruction as nausea and vomiting (Table 1). Ten patients presented with emergencies such as obscure overt gastrointestinal bleed (6) or bowel obstruction (4). Of the four patients that had obstruction two had lymphomas and two had gastrointestinal tumors.

Table no: 1: Presentation at the tumors of small bowel

\begin{tabular}{|l|l|l|}
\hline Clinical Symptoms & No of patients & Percentage \\
\hline Abdominal pain & $20 / 26$ & $(76.92 \%)$ \\
\hline Obscure GI Bleed & $17 / 26$ & $(65.38 \%)$ \\
Occult & $10 / 17$ & $(58.82 \%)$ \\
Overt & $7 / 17$ & $(41.17 \%)$ \\
\hline Weight loss & $12 / 26$ & $(46.15 \%)$ \\
\hline Intestinal obstruction & $10 / 26$ & $(38.46 \%)$ \\
\hline
\end{tabular}

Majority of the small bowel tumor (65\%) presented with obscure GI bleed. All patients had two rounds of upper and lower GI Endoscopies and then were subjected to either capsule endoscopy (5 patients) or CT enterocalysis (21). After the availability of 64 slices at our institute CT enterocalysis was used as next investigation of choice. CT enterocalysis or enterography was found to very effective in picking up small bowel lesions in another study conducted at our hospital on obscure gastrointestinal bleed. In present study 18/21 that had this investigation done a diagnosis could be clinched. Other patients had capsule endoscopy done before the availability of 64 Slice CT at our hospital or was investigated out side the hospital

All patients were subjected to surgery with a curative intent. R0 resection could be achieved in 23/26 patients (88.46\%). Two patients had R1 resection and one case of adeno carcinoma in the setting of Peutz Jeghers syndrome had only an ileoileal bypass.

Duodenal location was least common. Two of the four duodenal tumors were treated with Pylorus preserving pancreatico- duodenectomy. All others were treated with $10 \mathrm{~cm}$ margins and a wide mesentery. These tumors were almost equally common in Jejunum and ileum (Table2\&3).

Table no: 2 : Type of surgery performed according to disease distribution

\begin{tabular}{|l|l|l|}
\hline Type of operation & No: of patients & Percentage \\
\hline PP Whipples & 2 & $7.69 \%$ \\
\hline Duodenal excision & 2 & $7.69 \%$ \\
\hline Jejunal excision & 10 & $38.46 \%$ \\
\hline Ileal excision & 11 & $42.30 \%$ \\
\hline Ileoileal bypass & 1 & $3.84 \%$ \\
\hline
\end{tabular}


Histopathological evaluation revealed GIST and adenocarcinoma to be the commonest variety of small bowel lesions followed by lymphoma and neuroendocrine tumors (Table 3) (Fig 1-4).

Postoperatively there was no mortality and an acceptable morbidity of $2 / 26(7.6 \%)$ that included one case of prolonged ileus and one case of deep vein thrombosis.
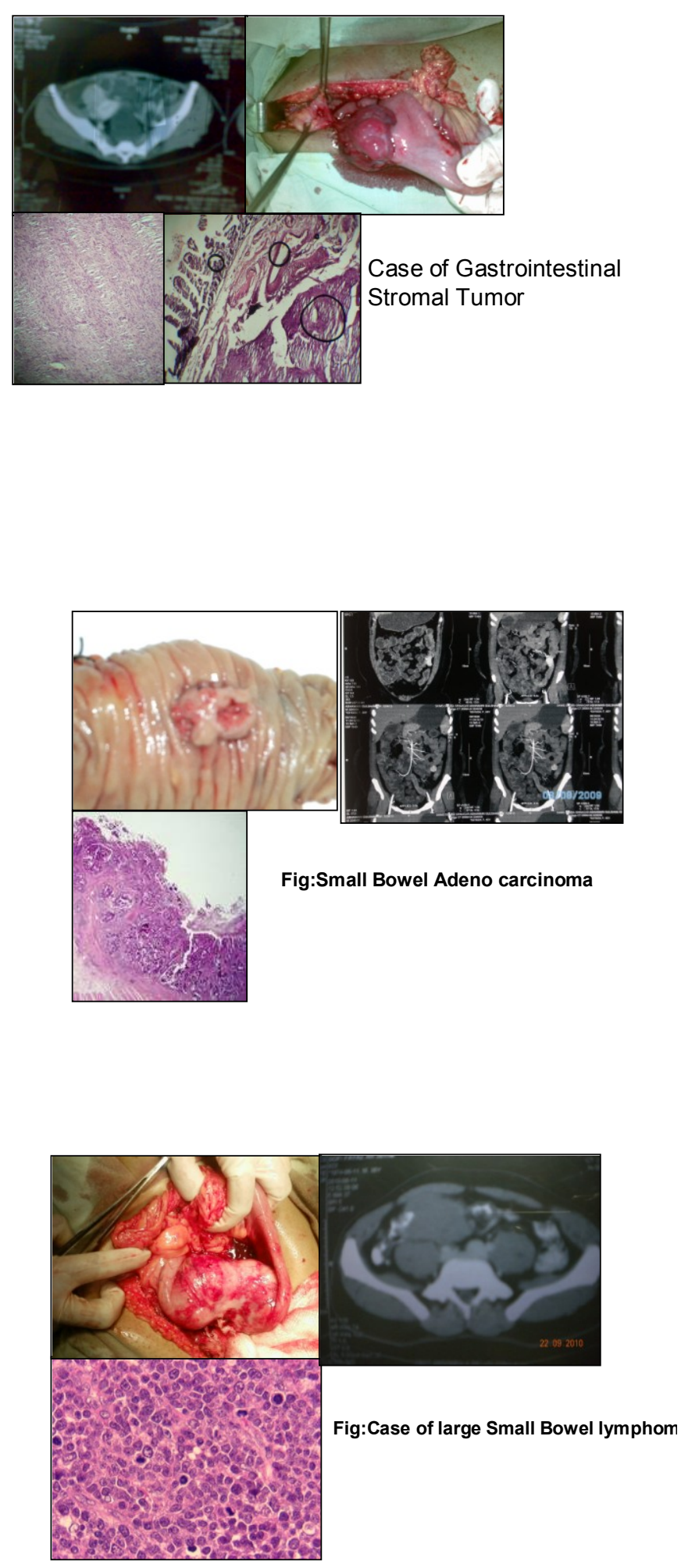


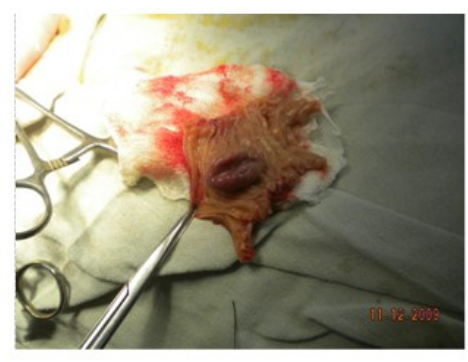

Fig:Excised proximal jejunal tumor(carcinoid) who presented as obscure GI ble

Table no: 3 : Tumor Histopathological diagnosis and distribution

\begin{tabular}{|l|l|l|l|l|}
\hline Tumor pathology & $\begin{array}{l}\text { Duodenum } \\
(4)\end{array}$ & $\begin{array}{l}\text { Jejunum } \\
(11)\end{array}$ & $\begin{array}{l}\text { Ileum } \\
(11)\end{array}$ & $\begin{array}{l}\text { Total :(percent) } \\
(26)\end{array}$ \\
\hline GIST & 1 & 6 & 4 & $11 / 26(42.30 \%)$ \\
\hline Adeno ca & 2 & 3 & 3 & $8 / 26(30.76 \%)$ \\
\hline lymphoma & 0 & 2 & 2 & $4 / 26(15.38 \%)$ \\
\hline Neuroend tumor & 1 & 0 & 2 & $3 / 26(10.71 \%)$ \\
\hline
\end{tabular}

On long term follow up the survival was highest at three years in ileal tumors when compared to jejunal and duodenal tumors. It was also observed that histopathological diagnosis of GIST had more favorable survival at 3 years and adenocarcinoma had the least survival. There were 20 of 26 patients surviving at the end of 3 years making up 76.92\% 3 year survival. Overall the disease prognosis of GIST was favorable and adenocarcinoma was dismal (Table 4\&5).

Table no: 4: Long term follow up of Small bowel tumors in relation to location

\begin{tabular}{|l|l|l|}
\hline Tumor location & Patients surviving at 1 year & Patients surviving at 3years \\
\hline Duodenum & $2 / 4(50 \%)$ & $1 / 4(25 \%)$ \\
\hline Jejunum & $10 / 11(90.91 \%)$ & $9 / 11(81.81 \%)$ \\
\hline Ileum & $10 / 11(90.91 \%)$ & $10 / 11(90.91 \%)$ \\
Total survivors & $22 / 26(84.61 \%)$ & $20 / 26(76.92 \%)$ \\
\hline
\end{tabular}

Table no: 5: Long term follow up of small bowel tumor in relation to pathological diagnosis

\begin{tabular}{|l|l|l|}
\hline Tumor Pathology & One year survival & Three year survival \\
\hline Adenoca & $5 / 8(62.5 \%)$ & $4 / 8(50 \%)$ \\
\hline Lymphoma & $3 / 4(75 \%)$ & $3 / 4(75 \%)$ \\
\hline Neuro end tumor & $3 / 3(100 \%)$ & $2 / 3(66.66 \%)$ \\
\hline GIST & $11 / 11(100 \%)$ & $10 / 11(90.90 \%)$ \\
Total survivors & $22 / 26(84.61 \%)$ & $20 / 26(76.92 \%)$ \\
\hline
\end{tabular}

\section{Discussion:}

Small bowel may be involved in wide variety of tumors, both malignant and benign. We have reviewed the scenario of malignant small bowel tumors in a tertiary health care centre. Malignant tumors include adenocarcinomas, carcinoids, sarcomas, and lymphomas, while benign lesions include adenomas, leiomyomas, lipomas, and hamartomas.

The clinical manifestations of small intestinal tumors are generally vague and nonspecific. Nonspecific and vague symptoms include pain, nausea, and vomiting related to partial or complete bowel obstruction. Bleeding or perforation may occur and OGIB is the most common presentation of small intestinal tumors ${ }^{5}$. In patients presenting OGIB, small intestinal tumors are diagnosed in around $6 \%{ }^{6}$ Weight loss, which if occurs should alert the physician to search for underlying organic causes and lead to a more thorough diagnostic workup, specially in patients aged over (older than) 50-years-old.

Though the advance of CT technology has allowed data acquisition over the entire abdomen in thin slices within one breath-hold, leading to fewer peristaltic and breathing artifacts, conventional CT scans detect only large size of intestinal tumors, at least over $1.0 \mathrm{~cm}$ in diameter. CT enteroclysis (CTE) is used to perform enhanced CT scanning and image post-processing after the small bowel is distended by administrating a high volume of contrast medium orally or via a nasojejunal catheter. It is easy to perform and produce reduced discomfort and less complication. The best merit for CTE lies in the fact that it not only provides the picture of the luminal side of mucosa but also provide the information about the changes in adjacent and related organs and 
structures. In view of easy and safe procedure, CTE become the primary choice for the detection and localization of small intestinal tumors ${ }^{7}$. Considering the literatures in the past were written before the introduction of newer small intestinal investigation modalities, the real incidence of small intestinal tumor may be higher than we had known ${ }^{8}$. Modalities like MRI and Capsule endoscopy are also the other investigations of choice, but authors believe that $\mathrm{CT}$ enterocalysis is more affordable and patient compliant investigation in our set up

The most common histologic types of malignant tumors of the small intestine in population-based registry data from the Surveillance, Epidemiology and End Results (SEER) program of the National Cancer Institute were adenocarcinoma, 45 percent; carcinoid, 29 percent; lymphoma, 16 percent; and sarcoma10 percent.

Adenocarcinoma is the most common primary malignancy of the small bowel and accounts for $40 \%$ of cancers

${ }^{9}$. The predominant location of adenocarcinoma is the Duodenum and proximal jejunum, with the incidence decreasing distally. An increased incidence of small bowel adenocarcinoma has been described in patients affected by familiarly adenomatous polyposis and lynch syndrome.

Surgical resection remains the cornerstone of therapy for these malignancies and studies have shown significantly longer median survival for the resected group ${ }^{10}$.Much of the information on the effectiveness of surgery for early GI lymphoma is based on limited, retrospective reviews that do not specifically compare primary surgical therapy with primary medical management are either chemotherapy, radiotherapy, or radio chemotherapy. Overall low prevalence of this disease, few clinical trial of chemotherapy have been conducted and a variety of chemotherapeutic agents used to treat adenocarcinoma of the small bowel, no standard chemotherapy regimen exists for this disease and usually a trend to follow as per large protocols prevail.

Carcinoid is the second most common malignancy, accounting for approximately $20-25 \%$ of all small bowel tumors, though its frequency has been recently increasing ${ }^{11}$. Much is known about carcinoids in the general population,including the relation of metastatic potential to size and site of origin and the generally favorable 5 -year survival rate ${ }^{12}$.

GISTs and leiomyosarcomas can have a similar morphologic appearance, but their distinction is important because treatment, particularly in the setting of advanced disease, differs markedly. More than 80 percent of GISTs have activating mutations in the KIT proto-oncogene, which leads to constitutive expression of c-kit, a tyrosine kinase receptor. Expression of c-kit can be identified by immunohistochemical staining (IHC).Enblock resection is again the primary treatment of GISTs. Unlike adenocarcinoma and carcinoid, sarcomas infrequently metastasize to regional mesenteric lymph nodes, and routine mesenteric lymphadenectomy is neither necessary nor beneficial. Excellent improvements in tumor control occurred with the recognition that mutational activation of KIT or a second receptor tyrosine kinase, platelet derived growth factor receptor-alpha (PDGFRA), stimulated the growth of these cancer cells, and that growth could be inhibited with orally active small molecule tyrosine kinase inhibitors (TKIs) that is Imitanib. CT scan is proven to be a very useful investigation in the diagnosis and follow up this small bowel tumor ${ }^{13}$.

Primary gastrointestinal (GI) lymphomas can be operationally defined as lymphomas in which the main bulk of disease is confined to the GI tract. The most common subtypes of primary small intestinal non-Hodgkin lymphoma are diffuse large-B cell lymphoma, enteropathy-associated T-cell intestinal lymphoma, extranodal marginal zone B-cell lymphoma of mucosa-associated lymphoid tissue (MALT), mantle cell lymphoma, and Burkitt and Burkitt-like lymphoma . Ileum is the most common site (60-65\%) involved, followed by jejunum $(20-25 \%)$, duodenum $(6-8 \%)$ and other sites $(8-9 \%){ }^{14}$.The treatment approach to a small bowel lymphoma generally parallels the standard treatment approach for that histological subtype of lymphoma arising in nodal regions. The outcomes following surgery alone are poor and systemic chemotherapy is indicated, even if resection is complete. Intestinal lymphomas differ significantly from their gastric counterpart,

not only in pathology, but also with regard to clinical features, management and prognosis. Our experience confirm the efficacy of the surgery chemotherapy combination in obtaining a good

remission rate for localized early primary intestinal

lymphoma ${ }^{15}$.

\section{Conclusion:}

A good number of cases of obscure GI bleed may be due to small bowel tumors. CT enterography is a promising investigation for small bowel evaluation. If diagnosed timely with high index of suspicion that small bowel tumors do exists the overall prognosis is not that dismal. 


\section{References:}

[1]. Cheung DY Choi MG. Current Advance in Small Bowel Tumors. Clin Endosc 2011;44:13-21

[2]. Bilimoria KY, Bentrem DJ, Wayne JD, Ko CY, Bennett CL, Talamonti MS. Small bowel cancer in the United States: changes in epidemiology, treatment, and survival over the last 20 years. Ann Surg 2009;249:63-71.

[3]. Cheung DY, Lee IS, Chang DK, et al. Capsule endoscopy in small bowel tumors: a multicenter Korean study. J Gastroenterol Hepatol 2010;25:1079-1086

[4]. Pilleul et al. Possible Small - Bowel neoplasms : Contrast enhanced Multidetector CT enterocalysis. Radiology:2006;241:3.

[5]. Gore RM. Small bowel cancer. Clinical and pathologic features. Radiol Clin North Am 1997;35:351-360.

[6]. Ohmiya N, Yano T, Yamamoto H, et al. Diagnosis and treatment of obscure GI bleeding at double balloon endoscopy. Gastrointest Endosc 2007;66(3 Suppl).

[7]. Kamaoui I, De-Luca V, Ficarelli S, Mennesson N, Lombard-Bohas C, Pilleul F. Value of CT enteroclysis in suspected small-bowel carcinoid tumors. AJR Am J Roentgenol 2010;194:629-633

[8]. Macari M, Megibow AJ, Balthazar EJ. A pattern approach to the abnormal small bowel: observations at MDCT and CT enterography. AJR Am J Roentgenol 2007;188:1344-55.

[9]. M Anzidei, A Napoli, C Zini et al. Malignant tumours of the small intestine: a review of histopathology, multidetector CT and MRI aspects .The British Journal of Radiology, August 2011

[10]. J. H. Donohue, Malignant tumors of the small bowel, Surgical Oncology, vol. 3, no. 2, pp. 61-68, 1994.

[11]. Marshall JB, Bodnarchuk G. Carcinoid tumors of the gut.Our experience over three decades and review of the literature. J Clin Gastroenterol 1993;16:123-9.

[12]. Caplin ME, Buscombe JR, Hilson AJ, Jones AL, Watkinson AF, Burroughs AK. Carcinoid tumour. Lancet 1998;352:799-805.

[13]. Hong X, Choi H, Loyer EM, Benjamin RS, Trent JC, Charnsangavej C. Gastrointestinal Stromal tumor: role of CT in diagnosis and in response evaluation and surveillance after treatment with Imatinib. Radiographics 2006;26: 481-95.

[14]. Schottenfeld D, Beebe-Dimmer JL, Vigneau FD. The epidemiology and pathogenesis of neoplasia in the small intestine. Ann Epidemiol

[15]. Di Sario JA, Burt RW, Vargas H, McWhorter WP. Small bowel cancer:epidemiological and clinical characteristics from a population based registry. Am J Gastroenterol 1994; 89:699-701 